\title{
Confidence Intervals on Implicit Association Test Scores Are Really Rather Large
}

\author{
Colin Klein \\ School of Philosophy \\ The Australian National University \\ colin.klein@anu.edu.au
}

\begin{abstract}
Implicit association test scores are presented as point estimates. Unusually for a psychological measure, individual scores are not tested for statistical significance in standard practice. This study estimates individual confidence intervals for a large dataset of IAT scores. The intervals are large. Only half of scores are significantly different from zero. This result raises theoretical concerns about standard interpretations of the IAT.

Keywords: Implicit Bias; IAT; Confidence Intervals
\end{abstract}

Author note June 3, 2020: This result strikes me as important, and it is also robust against many ways of calculating CIs. I am not a statistician, however, and doing this in a way that would satisfy statisticians remains out of my reach. This is probably an abandoned project, but if you have thoughts or ideas please do get in touch.

The Implicit Association Test (IAT) remains controversial. At issue is individual-level interpretations of the IAT $D$ score. It is commonly assumed that an individual's $D$ reflects something like a tacit attitude or trait. The home page of Project Implicit asks users to register "to find out your implicit associations about race, gender, sexual orientation, and other topics!" Presumably this is because an individual ought to be interested in their score, and may potentially gain moral obligations upon learning it. Philosophical treatments of IAT scores similarly focus on individual-level ethical consequences [1]. Conversely, critical examinations of the IAT focus on individual-level utility, with skeptics arguing that that $D$ scores are unstable and account for only a small amount of variance in individual actions [2].

Reporting of the IAT $D$ score is peculiar among psychological measurements. $D$ is a scaled estimate of the difference in mean reaction times in in stereotype-congruent and -incongruent matching tasks. It is standard practice in psychology to test differences in means for statistical significance, and ideally to report them with confidence intervals. Yet $D$ is reported-to individuals and in the literature - as a point estimate, without any measure of uncertainty.

The uncertainty around $D$ is critical for its interpretation. $D$ is often combined with precise cutoffs to bin test-takers into 'low', 'medium', or 'high' implicit bias. If one person is ranked 'low' and another is ranked 'high', one might naturally conclude that they differ in the amount of implicit bias. Yet this is a sensible conclusion only if confidence intervals on $D$ are narrow.

Note that this is a distinct (though related) problem to the test-retest reliability of the IAT. It is also orthogonal to questions about what the IAT actually measures. Whatever $D$ is meant to track, there is a further question about whether a particular $D$ score is statistically significant. Confidence intervals on $D$ would also provide this information.

Despite the practical and theoretical utility of confidence intervals on $D$, the literature appears 
to contain no systematic attempt to estimate them. This is likely because the calculation of $D$ is itself somewhat subtle, and because readily available scripts pre-compute $D$ directly.

This report details the estimation of confidence intervals using an open dataset from five years of Project Implicit. It shows that that confidence intervals on $D$ are often large. Indeed, they are large enough that we should reconsider regularly attributing properties to individuals on the basis of the IAT.

\section{Methods}

Data used was from the OSF repository for Project Implicit (https://osf.io/y9hiq/), using the five years of Race IAT datasets spanning 2010-2104. To avoid order effects, the dataset was filtered to include only completed trials where the stereotype-congruent blocks (i.e. 'white' paired with 'good') occurred first. This left 458852 records.

The IAT measures reaction times to categorization tasks in blocks of stereotype-incongruent and stereotype-congruent pairings. The difference in mean reaction times between blocks is divided by the combined standard deviation for the reaction times across both conditions [3]. In the dataset in question, there are two $D$ scores calculated - one for the 'practice' blocks of 20 trials each and on for the 'test' blocks of 40 trials. Using the trial block numbers, we refer to these as $D_{36}$ and $D_{47}$ respectively. In earlier scoring algorithms $D_{36}$ was discarded. In more modern ones (including within the period of the OSF dataset ) both pairs of blocks are instead averaged. Hence $D=\left(D_{36}+D_{47}\right) / 2$.

For a pair of blocks $a$ and $b, D_{a b}=\frac{\bar{m}_{b}-\bar{m}_{a}}{s_{a b}}$, where $s_{a b}$ is the combined standard deviation for the $a$ and $b$ blocks. Note that combined SD is distinct from the more familiar pooled standard deviation used in calculating Cohen's $d$. Combined SD has the convenient property that it bounds $D$ to $[-2,2][4$, fn 2].

Combined SD can be recovered from the reported data using $s_{a b}=$

$$
\sqrt{\frac{\left(n_{a}-1\right) s_{a}^{2}+\left(n_{b}-1\right) s_{b}^{2}+n_{a}\left(\bar{m}_{a}-\bar{m}_{a b}\right)^{2}+n_{b}\left(\bar{m}_{b}-\bar{m}_{a b}\right)^{2}}{n_{a}+n_{b}-1}}
$$

where $\bar{m}_{x}, s_{x}, n_{x}$ are the mean, standard deviation, and number of observations for block $x$.

The confidence interval on a $D$ score should be a function of the confidence intervals on $D_{36}$ and $D_{47}$. To simplify the problem of calculating $D_{a b}$, we make two assumptions: that the difference between means across trial blocks follows a t-distribution, and that $s_{a b}$ is constant across the confidence interval. Under these assumptions the problem reduces to calculating the confidence interval on the mean and dividing this by the combined SD. Given the t-distribution $t$, the overall chosen two-tailed significance level $\alpha$ (here .05 unless otherwise noted), and the Welch-Satterthwaite approximation for degrees of freedom $\nu$, an individual $D_{a b}$ has a confidence interval of $D_{a b} \pm r_{D a b}$, where

$$
r_{D a b}=\frac{t_{\nu, \frac{\alpha}{2}} \sqrt{\frac{s_{a}^{2}}{n_{a}}+\frac{s_{b}^{2}}{n_{b}}}}{s_{a b}}
$$

To combine confidence intervals, we use the method proposed by [5]. Given lower and upper 
(a)

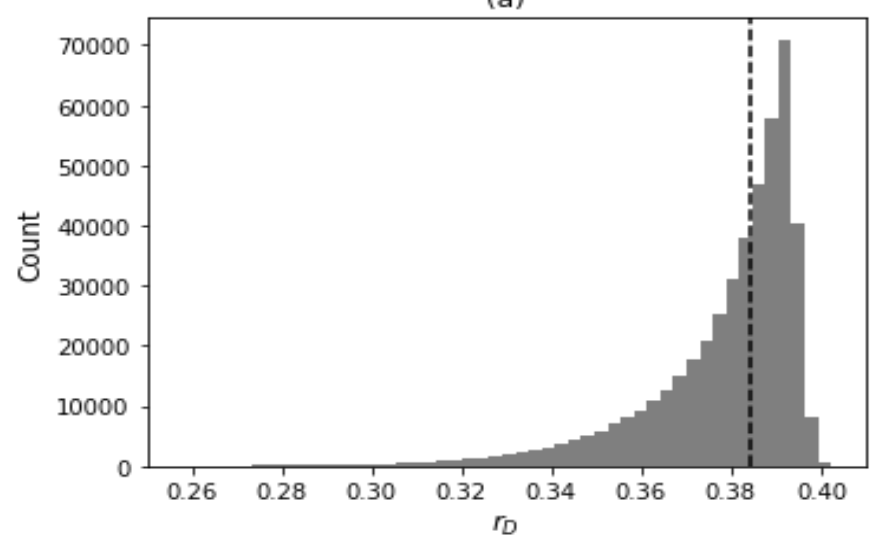

(b)

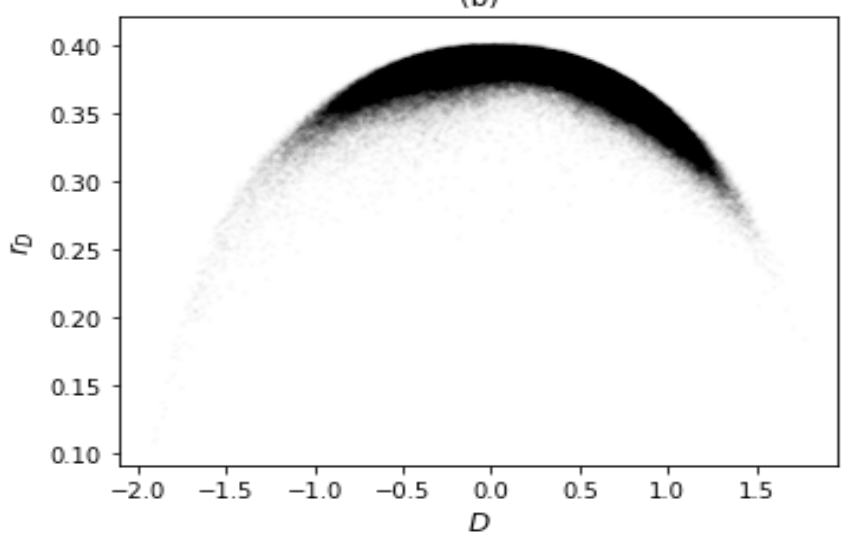

Figure 1: (a) distribution of $r_{D}$. Line at median $=0.38$. (b) scatterplot of $D$ versus $r_{D}$

bounds $\left[l_{36}, u_{36}\right]$ and $\left[l_{47}, u_{47}\right]$ we calculate lower and upper bounds $[L, D]$ :

$$
\begin{aligned}
L & =\frac{D_{47}+D_{36}-\sqrt{\left.\left(D_{36}-l_{36}\right)^{2}+\left(D_{47}-l_{47}\right)^{2}\right)}}{2} \\
U & =\frac{D_{47}+D_{36}+\sqrt{\left.\left(u_{36}-D_{36}\right)^{2}+\left(u_{47}-D_{47}\right)^{2}\right)}}{2}
\end{aligned}
$$

Then $r_{D}=\frac{U-L}{2}$, and the $95 \%$ CI is $D \pm r_{D}$.

To round out the picture, simple t-tests for independent samples were done on the mean reaction time differences in both the 3 vs 6 and 4 vs 7 blocks, again at $\alpha=0.05$ (uncorrected).

\section{Results}

Confidence intervals on $D$ are relatively large. Values of $r_{D}$ ranged from 0.11 to 0.40 , with a median of 0.38. Figure 1a shows that the distribution of $r_{D}$ is skewed. As $D$ is bounded in $[-2,2], r_{D}$ must be small for larger $D$ s. Figure $1 \mathrm{~b}$ illustrates the dependence.

A score is statistically significant when its confidence interval does not include 0 . Of $D$ scores in the dataset, $49.8 \%$ meet this criterion. So for slightly more than half of the participants, the null hypothesis that $D$ does not differ from 0 could not be rejected at an alpha of 0.05 . (uncorrected).

Comparison of statistical significance for individual trial blocks gives broadly consistent results. Only one pair of trial blocks reached significance for $45 \%$ of subjects, and for $22 \%$ neither pair reached significance. Broadly speaking, when $|D| \leq 0.5$, at least one trial block usually failed to reach significance.

\section{Discussion}

IAT $D$ scores are usually presented as point estimates. Yet the estimated confidence interval for $D$ is usually large. The median $95 \%$ confidence interval in the dataset spans a range of 0.76 points: i.e. $20 \%$ of the possible range of $D$ scores. In about half of the cases, the confidence interval is large enough that the null hypothesis cannot be rejected. 
The confidence intervals also span theoretically significant cutoffs. Using the standard cutoffs for 'slight' (.15), 'moderate' (.35) and 'strong' (.65) effects, an individual with a $D$ score of 0.4 would qualify as having moderate bias. Yet the median confidence interval would cover the range from no bias at all to strong bias. In the dataset, $22.6 \%$ of participants have $|D|>0.65$, the cutoff for 'strong' bias. This should be the most troubling individual result. Yet for a full $82 \%$ of those participants, the confidence interval spans below the 0.65 cutoff; $28 \%$ of those have confidence intervals that extend into the 'slight' bias range.

Categorizations of $D$ scores are somewhat arbitrary. The point is more general. Half of $D$ scores in a very large dataset do not differ significantly from zero. Insofar as individual $D$ scores bring differing ethical obligations, it matters whether $D$ scores can be confidently said to differ. The breadth of confidence intervals, particularly among individuals with the most common $D$ scores, casts doubt on that possibility.

This result suggests a number of potential impacts on research. Giving individuals precise scores without a standard $95 \%$ CI should be discouraged. So should categorizations which rely on binning scores with precise cutoffs. This is most important for the public reception of IAT research, as this is an area where public uptake has been especially high.

Ongoing debates about the replicability and behavioral effects of the IAT should also take into account the non-uniformity of $r_{D}$. Most discussions about (e.g.) test-retest reliability of the IAT implicitly assume that variability is uncorrelated with $|D|$. It is possible that a better picture of both the strengths and the weaknesses of the IAT would emerge if it incorporated the expected variability in individual measurements. One also increase the number of trials that individuals undergo, which should reduce the estimated confidence intervals. However, given the prevalence of this particular way of conducting and scoring the IAT, changes in procedure risk fragmenting empirical results.

Our research suggests that confidence intervals on $D$ could easily be calculated and presented to participants. It also gives a straightforward procedure for doing so. However, we suggest that reflection upon the desirability of doing this might be in order. One lesson strongly suggested is that IATs are simply not appropriate as individual diagnostic measures, in either scientific or informal contexts.

Certainly, when a $D$ score fails to reach significance, the correct response would seem to be to withhold judgment altogether, rather than present results with faux precision (either as point estimates or as confidence intervals). At the very least, one should not present scores where the components do not reach significance. The results from simple t-tests individual trial blocks suggests that nearly a quarter of the time, mean differences in reaction times for individual trial blocks do not reach significance on either pair of blocks. If caution is extended to cases where differences in reaction times do not reach significance in at least one pair of blocks, then we ought to remain quiet about two thirds of participants.

Such might be beneficial in some respects. It is an open question whether the remaining subjects would display more robust linkages between implicit bias and behaviour. Yet the social impact and significance of the IAT would look quite different if between half and twothirds of participants could not confidently be told that they have any implicit bias at all — as, indeed, seems to be the case.

On a more upbeat note, IAT scores might still be useful in aggregate. Even noisy individual scores can reveal interesting patterns at the group level. There has been a recent revival of the idea that IAT is more useful as an measure of systematic bias, rather than as a measure of individual differences [6]. On this view, aggregate IAT measures may function as a "barometer of inequality" [7] which reflects a combination of social and structural factors alongside both 
stable and transient individual differences. Losing confidence in individual scores might have the salutary effect of focusing attention on the large, undeniable inequalities which remain a feature of contemporary society.

\section{References}

[1] Holroyd J, Scaife R, Stafford T (2017) What is implicit bias? Philosophy Compass 12(10):e12437.

[2] Machery E (2017) Do indirect measures of biases measure traits or situations? Psychological Inquiry 28(4):288-291.

[3] Greenwald AG, Nosek BA, Banaji MR (2003) Understanding and using the implicit association test: I. an improved scoring algorithm. Journal of personality and social psychology 85(2):197-216.

[4] Nosek BA, Sriram N (2007) Faulty assumptions: a comment on Blanton, Jaccard, Gonzales, and Christie (2006). Journal of Experimental Social Psychology 43(3):393-398.

[5] Donner A, Zou G (2012) Closed-form confidence intervals for functions of the normal mean and standard deviation. Statistical Methods in Medical Research 21(4):347-359.

[6] Payne BK, Vuletich HA, Lundberg KB (2017) The bias of crowds: How implicit bias bridges personal and systemic prejudice. Psychological Inquiry 28(4):233-248.

[7] Daumeyer NM, Rucker JM, Richeson JA (2017) Thinking structurally about implicit bias: Some peril, lots of promise. Psychological Inquiry 28(4):258-261. 